\title{
Aplicabilidad del yoga como recurso terapéutico, junto a las mujeres en el proceso de deshabituación del diazepam.
}

\section{Applicability that yoga how therapeutic use, with women on the process of reducing the doses of diazepam medication.}

Clarissa Dantas de Carvalho ${ }^{\mathrm{i}}$

Nágela Maria da Silva Correia ${ }^{\text {ii }}$

\section{Resumen}

El presente artículo analiza las contribuciones que el yoga puede traer a las mujeres que se encuentran en proceso de deshabituación de Diazepam. Fueron descritas las vivencias de esas mujeres y más específicamente se evalúa el modo como ellas confrontan las situaciones de su estar cotidiano, tanto sobre el efecto de los medicamentos, como también sobre los efectos de la práctica continuada de técnicas de yoga. La investigación fue desarrollada en el Centro de Atención Psicosocial (CAPS) de Maracanaú en el Estado de Ceará - Brasil. Los sujetos de la investigación fueron dos mujeres que estaban en proceso de deshabituación de Diazepam. Se utilizó como método de recolección de datos, al inicio, una anamnesis, y entrevistas semiestructuradas, al medio y al final de la investigación. Los datos fueron analizados a través de la técnica de análisis del discurso. Los resultados obtenidos con la investigación consideraron los beneficios de la práctica del yoga en la vida de las participantes y la posibilidad de la disminución de la ansiedad y el aumento del equilibrio emocional. Fue constatado que las prácticas de yoga condujeron a las participantes a una mejor autopercepción y a la concientización de la dependencia al medicamento. Hubo una reducción significativa en el uso del tranquilizante y hasta la interrupción total por parte de las participantes del estudio en cuestión. Además, se valida la necesidad de una transformación en la conducta de los profesionales de salud, que tratan no solamente las mujeres usuarias del Diazepam, sino también a las mujeres en general.

Palabras Claves: mujeres, deshabituación, diazepam, yoga.

\footnotetext{
${ }^{i}$ Bachiller en Terapia Ocupacional (UNIFOR) - CCS. cla_to@hotmail.com Tel. (55) 8530915459

${ }^{i i}$ Master en Psicología por la Universidad de Fortaleza (UNIFOR) - CCS. Av. Washington Soares

1321, Barrio Edson Queiroz Tel. (55) 8530915459
} 


\begin{abstract}
This paper analyses the contributions that yoga can bring to women who are in the process of Diazepam cessation. Were described the experiences of these women and more specifically it is evaluated the way they confront everyday situations, both on the effects of medicines as well as on the effects of yoga techniques. Research was carried out at the Psicosocial Care Center, in Maracanaú, Ceará -CAPS- Brazil . Subjects in this study were two women in the process of Diazepam cessation. Data collection methods were, anamnesis at the beginning, and the semi-structured interviews in the middle and at the end. Data analysis was carried out through Discourse Analysis technique, Results obtained with the study considered the benefits of yoga practice in participant's life, such as decrease of anxiety and increase of emotional balance. It was found that yoga practices led participants to a better self-perception and awareness that they were dependant of medicines. There was a significant reduction on the use of Diazepam and also a total cessation by some participants in the study. Furthermore, the need for a transformation in health professional's behavior is validated, by treating not only women who use diazepam, but also women in general.
\end{abstract}

Key Words: Women, Cessation, Diazepam, Yoga. 


\section{Introducción}

El presente estudio busca reflexionar acerca del uso problemático de mujeres adictas al Diazepam y su espacio social, y analizar los efectos de la práctica de yoga como una técnica de bienestar corporal. Esperamos validar esta práctica como medio alternativo de tratamiento para el desuso del medicamento, a fin de restablecer la integridad de las intervenciones en sus aspectos bio-psico sociales.

La presente investigación busca también comprender cuales son los efectos nocivos que pudieran afectar a la salud de la mujer en la vida diaria; considerando las condiciones de vida como el factor inicial determinante en el proceso de salud - enfermedad.

Realizando un análisis cultural biológico sobre el proceso de somatización, en clínica de la salud mental, (tratada comúnmente como enfermedad de los nervios o neurosis) se hace una investigación sobre los factores culturales que llevan a las mujeres a ser calificadas de "nerviosas". Los hombres, por otro lado, tienen acceso a válvulas de escape socialmente aceptables como el uso del alcohol, uso de los espacios públicos, una mayor convivencia con los amigos, etc. Por otra parte, el hombre está menos expuesto a sobrecarga de actividades estresantes como el cuidado de los hijos y de la casa, atribución claramente femenina en muchas culturas. (1)

¿La actual red de asistencia ofrecida a la salud de la mujer está realmente capacitada para ayudarlas? Estudios realizados sobre los médicos que atienden a mujeres usuarias de benzodizepins constatan una falta de preparación y hasta una visión parcializada en el proceso de prescripción del medicamento. (2)

Tal deficiencia del sistema de salud se refleja en el uso de Diazepam (marca de benzodiazepina mas vendida actualmente) por parte de mujeres afiliadas al sistema público de salud en los municipios de Fortaleza/CE e Sobral/CE. La incidencia del uso de ese medicamento por mujeres es alarmante como muestra un levantamiento hecho en la cuidad de Fortaleza /CE junto con la farmacia del sistema público de salud, en donde el $72 \%$ de los consumidores eran mujeres. Tales datos fueron comprobados en Sobral /CE donde el 75\% de los consumidores de la única farmacia de la red pública eran también mujeres. Datos que merecen una reflexión de lo que está representando este tipo de práctica en la vida de las mujeres. (2)

Las benzodiazepinas afectan principalmente el sistema nervioso central y sus efectos farmacológicos varían entre la disminución de la ansiedad y de la impulsividad, causando sensación de sedación y sueño. Aun en dosis terapéuticas puede llevar a un estado de dependencia y si es ingerido por más de 6 meses y su uso es súbitamente interrumpido, se pueden observar efectos de abstinencia tales como irritabilidad, insomnio, sudoración, algias corporales y en casos extremos hasta convulsiones. (3)

Por tanto, prescindir del medicamento en la asistencia de salud en mujeres es imprescindible. No se trata de eliminar su uso, pues esto sería muy riesgoso, además de que en general, para 
este tipo de medicinas el procedimiento de disminución de su uso es un proceso lento. Debemos trabajar en la desmitificación de la panacea, que hasta hoy, como observa Foucault (1972), persiste en la sociedad occidental. Tal concepción es la que impide a las mujeres tener una real comprensión de su salud y enfermedad. (4)

Los servicios de salud generalmente se organizan para atender a la mujer solo en su ciclo gravídico - puerperal, o sea en asistencia pre-natal y de parto, siendo aún deficiente como aporte a la salud integral de la mujer. Advertimos que el sistema de salud desatiende las necesidades específicas de la mujer y está volcado solamente a los aspectos físicos y biológicos sin considerar su subjetividad.(2)

En contrapartida a los modelos que visan una intervención externa dicotómica sobre el paciente, se propone una práctica basada en la búsqueda del contenido espiritual, teniendo como herramienta la autoobservación y la comprensión del ser en su integridad, en un equilibrio entre cuerpo, mente y emociones. Yoga en sanscrito significa unión, aplicación, trabajo. Yoga seria por lo tanto, el medio y el fin en sí mismo. (5)

El yoga es el recurso terapéutico que actúa por medio de la recuperación perceptiva de corporeidad en el individuo. Utilizando las técnicas de yoga buscamos rescatar la subjetividad del paciente, además el yoga posibilita un nuevo modo de ser, una manera que trasciende los fenómenos efímeros viabilizando el reconocimiento de las infinitas posibilidades del ser y de estar en el mundo. (6)

Partiendo de esas premisas, el objetivo es analizar la aplicación del yoga como una medida intervencionista que pudiera ayudar a las mujeres en el proceso de deshabituarse del uso del medicamento paulatinamente en paralelo a la práctica del yoga.

Se presume que esta investigación será relevante no solamente en las prácticas de terapia ocupacional, sino para todas las personas que utilizan tranquilizantes. Se busca también ofrecer el conocimiento a estudiantes, a otros profesionales del área de la salud mental, así como personas de diferentes áreas que buscan esclarecimientos sobre el deshabituamiento de las benzodiazepinas, de este modo contribuiremos al enriquecimiento de nuevos saberes para la ciencia y la sociedad.

\section{Método}

Los objetivos que guiaron este estudio ayudaron a definir el método de sistematización de esta experiencia. La investigación se realizó principalmente a nivel empírico, siendo de tipo longitudinal (7), de naturaleza cualitativa (8), constituyéndose en el estilo investigación acción (9).

La investigación fue desarrollada en el Centro de Atención Psicosocial (CAPS), vinculada a la red SUS de atención, situado en el municipio de Maracanaú - CE. El estudio ocurrió entre agosto y octubre del 2008 con encuentros semanales en la tarde con dos días a la semana 
(martes y jueves), totalizando veintiséis sesiones. Cada sesión dispuso de la estructura básica de una clase de Yoga como práctica de posturas, ejercicios respiratorios y relajación, respetando las condiciones físicas de los participantes. (9)

Al inicio de la investigación contamos con seis participantes mujeres, todas usuarias de Diazepam y en proceso de deshabituación. Antes de finalizar la aplicación de las prácticas, abandonaron cuatro participantes. Dos de ellas debieron cuidar a familiares, la tercera emigró a otra cuidad y la ultima tuvo problemas con el horario de trabajo. De este modo serán analizados los datos de las dos participantes que permanecieron del inicio al fin del estudio. Ambas son amas de casa, casadas, con hijos y no practicaban ninguna actividad física. Los rangos etáreos de las participantes eran de 49 y 39 años respectivamente.

Las técnicas para la recolección de datos utilizados fueron: observación participante, diario de campo, entrevista para de ingreso para la práctica de yoga, preguntas pertinentes a la investigación y una entrevista semi-estructurada.

El trabajo de campo consta se las siguientes etapas:

- Sensibilización de los profesionales de la institución para la realización de la investigación, esclareciendo los objetivos y la importancia del equipo de profesionales a cargo (psiquiatras, enfermero, psicólogos, terapeuta ocupacional y servicio social).

- Selección de los participantes dentro de grupos terapéuticos que se desarrollan en la institución, tanto del sector de terapia ocupacional, de psicología y entre los que cuentan con tratamiento clínico con los psiquiatras. Se dan indicaciones a los profesionales a cargo de los grupos para que colaboren en la selección.

- Sensibilización para la inclusión de participantes en la investigación: reuniones con usuarias del servicio que cumplían con el perfil deseado, es decir, que ya estuviesen en el proceso de deshabituación de Diazepam, con la colaboración de terapeuta ocupacional y coordinadora interna del servicio de atención psicosocial.

- Organización para el inicio de las prácticas y entrevista inicial para el reconocimiento detallado del participante. Invitación consentida a participar.

- Aplicación de entrevista semi estructurada en el plazo medio de la investigación para el control situacional de la perspectiva de cada participante sobre la práctica y seguimiento diario en la deshabituación de Diazepam.

- Aplicación de entrevista semi estructurada al final de la investigación para sumar las impresiones de los participantes en cuanto a la práctica de yoga y comprobar el nivel de uso actual del medicamento.

La entrevista fue grabada con la debida autorización de los participantes. En seguida fueron transcritas totalizando el tiempo de sesenta minutos aproximadamente. De ese modo y en orden de alcanzar los objetivos propuestos el análisis de los datos, se efectúa la técnica de análisis de discurso que opera por medio de un análisis específico del lenguaje como espacio social de debate y conflicto. El análisis del discurso permite comprender el proceso de producción de los sentidos y su relación con la ideología y también da al analista, la posibilidad de establecer regularidades en el funcionamiento del discurso. (10) 
La investigación fue autorizada por el comité de ética - COETICA de la Universidad de Fortaleza conforme la autorización N ${ }^{\circ} 207 / 08$. Además estuvo respaldada por la resolución 196/96 del Consejo Nacional de Salud para la investigación y estudio con seres humanos, aclarando que la participación de los sujetos investigados sería voluntaria y que los mismos se podrían retirar arbitrariamente. Durante todas las etapas del estudio estuvo presente la cautela por parte de la investigadora, ofreciendo el debido respeto a la dignidad humana manteniendo en sigilo la confidencialidad de las identidades e informaciones de cada persona.

\section{Resultados y Discusión}

La recolección de datos ocurrió en tres momentos. En el primero de ellos, se obtuvo los datos de la entrevista de diagnóstico, luego se recabaron aquellos de la entrevista semi estructurada tanto en el plazo medio como al final de la investigación.

El propósito de la entrevista inicial es la obtención de información personal, condiciones generales de salud, información en torno a la cotidianeidad de cada una de las investigadas, agregando preguntas relativas al uso del medicamento. (11)

Primera pregunta: ¿Como es su día a día?

\begin{tabular}{|l|l|}
\hline Participante 1 & "Hago las cosas en casa y lloro sin motivo" \\
\hline Participante 2 & $\begin{array}{l}\text { "Mi día es atareado lleno de quehaceres domésticos, como cuidar } \\
\text { los hijos, la casa etc." }\end{array}$ \\
\hline
\end{tabular}

Con las respuestas obtenidas a la pregunta de arriba se procede al análisis del discurso de las participantes. Nótese el contingente de quehaceres domésticos entrelazados a sus funciones diarias. Además del papel asumido por tales mujeres, se observa un vacío para la comprensión de sus emociones. Queda claramente demostrado al señalar "lloro sin motivo".

Las mujeres tiene su día a día cargado de demandas y de actividades que no son consideradas al momento de la atención medica, el resultado es el uso de tranquilizantes para poder soportar las dificultades cotidianas. (11)

Segunda pregunta: ¿Cuales son los motivos que le llevaron al uso del Diazepam?

\begin{tabular}{|l|l|}
\hline Participante 1 & "Lloraba mucho, y no tenía un motivo para estar llorando". \\
\hline Participante 2 & $\begin{array}{l}\text { "No tuve una buena relación con el padre de mis hijos, sufría de } \\
\text { agresión verbal". }\end{array}$ \\
\hline
\end{tabular}

Con las respuestas obtenidas a la pregunta 2, se observa el grado de opresión a las condiciones relativas de la subjetividad de las mujeres y a su lugar en el espacio social. La exposición al uso del medicamento denuncia un elevado conformismo en relación a los 
problemas que en estricto rigor, serían del ámbito medioambiental y no necesariamente médicos.

Es posible que los ansiolíticos se transformen en un verdadero opio social, enmascarando los síntomas emocionales mientras el contexto social continua inalterado, o generalizando diferentes disturbios y desviando el diagnostico correcto de las dolencias. (11)

Tercera pregunta: ¿Cuándo inició el uso de Diazepam?

\begin{tabular}{|l|l|}
\hline Participante 1 & "Estaba con unos 15 años en el hospital de Maracanaú" \\
\hline Participante 2 & $\begin{array}{l}\text { "Hace algunos años sentía crisis nerviosas, estaba trémula, manos y } \\
\text { pies helados empecé a usarlo en el hospital de Maracanaú, hace 17 } \\
\text { años sin el uso correcto." }\end{array}$ \\
\hline
\end{tabular}

Dicho hallazgo, apunta a una realidad que es enfrentada por varias mujeres, principalmente al constatarse el largo periodo de uso del tranquilizante. Una de ellas, lo utilizó desde los 15 años y la otra desde los 17 años. Por tanto, se percibe la vulnerabilidad femenina al uso del medicamento, tal fragilidad es acrecentada por la falta de asistencia especifica de su reales necesidades, agravada por la carencia de orientación del uso del tranquilizante. (2)

Cuarta pregunta: ¿Como usted se sentía haciendo uso del medicamento?

\begin{tabular}{|l|l|}
\hline Participante 1 & "Yo me sentía relajada, me sentía, mas liviana." \\
\hline Participante 2 & $\begin{array}{l}\text { "Me sentía bien, pasaron el mareo, las nauseas y el dolor de } \\
\text { cabeza" }\end{array}$ \\
\hline
\end{tabular}

Las participantes de la investigación identifican como efecto benéfico del Diazepam, la relajación, y el cese de cualquier dolor "de cabeza, mareo o nauseas". Otorgando al medicamento, la atribución de eliminación de síntomas aflictivos, claramente provoca la dependencia al uso.

A continuación apuntaremos los datos recolectados en las entrevistas realizadas con las participantes para el registro de las técnicas de yoga como recurso terapéutico utilizado en el proceso de deshabituación de Diazepam. Aquí serán transcritas y analizadas las narraciones de mayor relevancia para la investigación.

\section{Pregunta}

En cuanto a las técnicas de Yoga de su preferencia ¿cuáles son sus impresiones después de su realización?: 


\begin{tabular}{|l|l|}
\hline Participante 1 & $\begin{array}{l}\text { "La respiración y el relajamiento es bueno, mucho mejor, me siento bien así, } \\
\text { calma, por lo menos así es después que comencé a hacer, me estoy sintiendo } \\
\text { bien, mucho mejor de mi ansiedad, más calma no sentí mas dolor, porque } \\
\text { eso aquí me hinchaba, sentía falta de aire es así que aquello era tan malo, } \\
\text { aquella ansiedad que no consigo explicar ahora, estoy bien, más calmada, } \\
\text { mejor " }\end{array}$ \\
\hline Participante 2 & $\begin{array}{l}\text { "Estiramiento, ¿no?, es porque siento la diferencia en la postura, en la } \\
\text { musculatura ¿no? Si tuviera un dolor siento que mejoraría." } \\
\text { "En la respiración ¿no?, me siento bien por causa de la ansiedad, quien tiene } \\
\text { problema así de ansiedad ¿no?, es así que mejora bastante sentí una } \\
\text { diferencia." }\end{array}$ \\
\hline
\end{tabular}

En las narraciones, se expresa el énfasis sobre la experimentación vivida al momento de la clase. Las propias participantes toman conciencia de la modificación del patrón físico/emocional con las prácticas de las técnicas de yoga elegidas.

El Yoga nos posibilita una concientización de nuestros procesos físicos, emocionales y mentales. La realización de las técnicas corresponde a una verdadera alquimia corporal, parten de una actitud diferenciada recurriendo a la inteligencia corporal, alineamiento, y respiración consciente que propicie una transformación en el organismo. (5)

La utilización de las técnicas de yoga en el día a día de las mujeres:

\begin{tabular}{|l|l|}
\hline Participante 1 & $\begin{array}{l}\text { "Yo haciendo las cosas de la familia me canso mucho, muchas veces mi } \\
\text { hijo me está molestando, me perturba ahí le digo ipara nene!, salgo para } \\
\text { mi cuarto cierro la puerta y hago la respiración." } \\
\text { "quedo tensa ¿no?, ahí uso aquel ejercicio y así mejora mucho mi } \\
\text { respiración. Me quedo más relajada, mejora mucho." }\end{array}$ \\
\hline Participante 2 & $\begin{array}{l}\text { "Solo cuando estoy en dificultades ¿no?, ni siempre, pero cuando tengo } \\
\text { un tiempito, me acuerdo de practicar esa cuestión de la respiración, } \\
\text { llamo a mis hijitas para hacer juntas ..." } \\
\text { "Es cuando siento que estoy preocupada cuando viene mi ansiedad, ahí } \\
\text { ya me voy preparando para hacer la respiración, así mejoro." }\end{array}$ \\
\hline
\end{tabular}

A partir del análisis narrativo se percibe el empleo de las técnicas de respiración para el alivio de dolores y angustia. Las técnicas son usadas como una herramienta practica de relajación para los acontecimientos cotidianos, como discusiones con los hijos o momentos de fragilidad al sentirse preocupadas. Instantes que podrían dejarlas más susceptibles al uso de Diazepan, cuentan ahora con el soporte de una práctica de ejercicios respiratorios.

En el Yoga la respiración es mucho más que un hecho fisiológico, es también psicológico y energético, siendo el único proceso doblemente voluntario e involuntario. Reconociendo la posibilidad de que la respiración llega a nuestra subjetividad, se puede observar la relación clara entre el ritmo respiratorio y cada cambio emocional. 
En la inquietud mental y emocional se observa la respiración acelerada. En tanto estemos más tranquilos física, mental, y emocionalmente se advierte una respiración más pausada. Así, con los ejercicios respiratorios voluntariamente controlados volvemos la respiración más lenta e inducimos la tranquilidad mental y emocional. (12)

Cambios obtenidos como consecuencia de la participación en el grupo.

\begin{tabular}{|l|l|}
\hline Participante 1 & $\begin{array}{l}\text { “...Estoy durmiendo mucho mejor. Ese ejercicio de respiración, me hace } \\
\text { sentir bien, ¿sabes?. No sé explicar pero estoy bien. Duermo mejor. A } \\
\text { veces digo: caramba Maria estoy durmiendo mucho, cosa que a las 5 de } \\
\text { la mañana ya estaba despierta, ahora estoy durmiendo bien, estoy mucho } \\
\text { mejor." }\end{array}$ \\
\hline Participante 2 & $\begin{array}{l}\text { "Si, porque estoy mucho más calma, no soy mucho de ser aquella } \\
\text { persona agresiva pero tengo mis momentos ¿no? y mejoré bastante, } \\
\text { tengo más calma en determinadas cosas." } \\
\text { "Por ejemplo hago las cosas con mucha calma. Pero cuando están los } \\
\text { hijos, las cosas se desordenan un poco así que me quedo más agitada. En } \\
\text { ese sentido, en mi manera de actuar mejoré porque en vez de quedarme } \\
\text { así, ya no les pego, converso con ellos. Fue una manera de ver como } \\
\text { resultaba bien". }\end{array}$ \\
\hline
\end{tabular}

Notamos en el discurso de las participantes una mejora significativa en la calidad del sueño, en sus relaciones familiares, habiendo una modificación de sus actitudes ante los acontecimientos. Queda de este modo un espacio para la reflexión sobre la mejor actitud a tomar.

Observamos que el Yoga empleado como actividad corporal es un importante recurso para una resignificación de esas mujeres en su contexto. Muchas veces las técnicas se presentan necesarias para desviar el foco de las participantes de las situaciones que representan una amenaza al equilibrio físico y emocional viabilizando una "apertura del espacio" dentro de uno mismo frente al momento. El Yoga enseña a centrar la atención de lo que sucede en el cuerpo, a sentir las emociones y a soltarse. Evidencia que nada es permanente y que los sentimientos no somos nosotros mismos. (13)

En consecuencia, lo que desencadena es una modificación en el día a día de estas mujeres. Luego, el cotidiano, se define como aquello que se realiza, como se realiza, con quien y donde y además como eso es apreciado por ellas y por los que le rodean. El Yoga es una técnica que induce a la introspección y también despierta el interés por el otro, estimulando la cooperación para una convivencia social más armónica y saludable. (6)

El Yoga está basado en la visión holística del ser y tener una salud física y mental como meta. Toda dolencia es apenas un reflejo de un desequilibrio interno, y, al establecernos en este equilibrio la salud retorna. (15) 
Deshabituación de Diazepam y contribuciones de la participación en el grupo de Yoga para este proceso.

\begin{tabular}{|l|l|}
\hline Participante 1 & $\begin{array}{l}\text { "Pare, ya no tomo mas, yo venía disminuyendo, tomaba de vez en } \\
\text { cuando, pero ahora no tomo." } \\
\text { "Bien mejor, porque el Diazepam que tomaba me hace quedar toda } \\
\text { adormecida, soñolienta, así es. No sé si me deja un poco deprimida y } \\
\text { sin él me siento otra persona, se siente uno mejor, ser uno misma." } \\
\text { "Mejoré así de todo un poco, en mi día a día, en la ansiedad, me siento } \\
\text { mejor, con más ganas, ¿me entiendes?. No me quedo mas llorando y } \\
\text { comiendo, solo vivía comiendo... y aun siento aquella ansiedad pero } \\
\text { no como sentía antes, antes sentía mucha, una ansiedad de mi Dios del } \\
\text { Cielo." }\end{array}$ \\
\hline Participante 2 & $\begin{array}{l}\text { "... busco tomar más en el periodo de menstruación la cuestión es que } \\
\text { cuando está faltando diez días antes y diez días después estoy bastante } \\
\text { irritada, siento mucho dolor de cabeza pero solo en ese periodo, } \\
\text { sacando ese periodo no, está haciendo la diferencia ya que solo tomo } \\
\text { en ese periodo, ahora hay diferencia porque yo tomaba de forma } \\
\text { constante. ¿me entiende? }\end{array}$ \\
\hline
\end{tabular}

Podemos observar en los discursos obtenidos de esas mujeres que su trayectoria en el proceso de deshabituación del Diazepam fue trazada conjuntamente con las prácticas de Yoga. Se constatan los beneficios de la práctica de Yoga en la vida de las participantes a partir del relato de la disminución de la ansiedad y de la alimentación compulsiva, además del aumento de la disposición al equilibrio emocional. Las técnicas posibilitaron aun más la auto percepción y la concientización de la posibilidad de la dependencia al medicamento. Queda evidenciada una reducción significativa y hasta el abandono del uso del tranquilizante en las participantes del estudio.

Constatamos en las participantes una nueva actitud. Eso significa una toma de conciencia en el sentido de entender y buscar soluciones a los propios problemas que las hacen padecer, volviéndose protagonistas de aquello que les acontece. (15)

Observamos entonces que la práctica de Yoga propone ayudar al cuerpo a liberarse de la carga toxica y volver a la condición de salud. Esta desintoxicación tan benéfica y generadora de salud actúa no solamente en el campo físico sino también en el campo energético, en las emociones, en los pensamientos y las convicciones más profundas del individuo. (16) Por lo tanto en este proceso la construcción, destrucción y reconstrucción del sujeto ocurre de forma continua. (17) De este modo podemos considerar lo que afirma Almeida:

"Corporeidad y la terapia ocupacional están relacionadas a un cuerpo en creación, que a cada nueva experiencia el cuerpo se remodela posibilitando nuevas percepciones del mundo y consecuentemente producir una fuerza política y transformadora. Todo nuevo cuerpo es un sujeto en el mundo."(18) 
Después de la experiencia descrita se puede verificar los beneficios del Yoga y pensar en la viabilidad de su uso como recurso terapéutico para la terapia ocupacional.

\section{CONSIDERACIONES FINALES}

El Diazepam es un tranquilizante que es utilizado de forma indiscriminada y que se ofrece como recurso para silenciar angustias y necesidades de las mujeres. El medicamento se volvió un apaciguador frente a las tensiones que existen en el universo femenino tanto que evita la reflexión de la mujer ante las situaciones de conflicto e imposibilita la revalidación del sujeto sobre si.

Podemos, a partir de esta investigación, considerar la práctica del Yoga como recurso terapéutico satisfactorio en el tratamiento de mujeres que se encuentran en el proceso de deshabituación de Diazepam. A través de las técnicas de Yoga, las mujeres experimentan sensaciones benéficas tanto en el cuerpo, como en las emociones y la mente. Tales beneficios no se limitaron al momento de nuestros encuentros, sino que se extendieron a su quehacer cotidiano. Constatamos modificaciones significativas en su modo de posicionarse ante situaciones y más directamente de su actitud en relación al medicamento.

Los aspectos analizados en este trabajo muestran la necesidad de direccionar acciones en las políticas públicas para la modificación de la conducta del profesional de salud junto a la mujer usuaria de Diazepam. Nótese la especial necesidad de un programa para mujeres a fin de esclarecer sobre la dependencia de las benzodiacepinas, y de apoyar un programa de intervención directa en el proceso de deshabituación del tranquilizante. Solamente a partir de esas modificaciones podrán las benzodiazepinas ser desapropiadas de su papel de muleta social.

\section{REFERENCIAS BIBLIOGRÁFICAS}

1.SILVEIRA M. O nervo cala, o nervo fala: a linguagem da doença. Rio de Janeiro: Editorial Fiocruz, 2004;39-43.

2.OLIVEIRA E. Saúde mental e mulheres: sobrevivência, sofrimento e dependência química. Sobral: Editorial Uva, 2000; 10 -55.

3.CARNEIRO M. Motivos e influencias do uso de benzodiazepínicos em mulheres: Estudo realizado em um CAPS no interior do Ceará. Saúde em debate..[periódico online] 2005;79(consultado em 12 de noviembre de 2008). Disponible en

URLhttp://cebes.iphotel.info/media/File/publicacoes/Rev\%20Saude\%20Debate/Saude\%20em \%20Debate_n70.pdf\#page $=109$.

4.CARVALHO, F. Dependência química em mulheres:um estudo sobre o consumo de medicamentos ansiolíticos no serviço publico de saúde de Natal/RN. Universidade federal do rio grande do norte.Programa de pós -graduação em psicologia.[monografia online].2001;2:10-17(consultado em 15 octubre2008).Disponibleen:http://bdtd.bczm.ufrn.br/tedesimplificado/tde_arquivos/1/TDE2006-04-27T000500Z-2/Publico/LuciaFC.pdf 
5.KUPFER P. Yoga prático. Florianópolis: Editorial Dharma,2001;33-35.

6.WIEZZER K. O yoga como estratégia de promoção à saúde: Relato de experiência em uma unidade de saúde da família em Blumenau. Dynamis Revista Tecno - Cientifica. 2004; 47, 27 43.

7.GIL, A. Métodos e técnicas de pesquisa social. São Paulo: Editorial Atlas, 1991,12-13.

8.MINAYO M. Pesquisa social: teoria, método e criatividade. $6^{\text {a }}$ ed. Petrópolis. Rio de Janeiro: Editorial Vozes;1994,25-38.

9.MEYER J. Usando métodos qualitativos na pesquisa-ação relacionada à saúde. In: Pope C. pesquisa qualitativa na atenção a saúde. $2^{\mathrm{a}}$ ed. Porto Alegre: Editorial Artmed;2005,11-12.

10.ORLANDI P. Análise de discurso, princípios e procedimentos. $2^{\mathrm{a}}$ ed. Campinas.São Paulo: Editorial Pontes; 2000, 23-25.

11.CARVALHO L. A mulher, seu médico e o psicotrópico: Redes de interfaces e a produção de subjetividade nos serviços de saúde. Interelações. [periódico online] 2003;2:915. (consultodo en 17 de novienbre de 2008).Disponible en URL:http://scielo.bvspsi.org.br/scielo.phppid=S141329072003000100003\&script=sci_arttext \&tlng=pt.

12.HERMÓGENES J. Auto Perfeição com o hatha yoga, $27^{\mathrm{a}}$ ed.Rio de Janeiro: Editorial Record;1988,45-60.

13.SPARROWE L. O livro de yoga e saúde para a mulher: Um manual de bem estar para a vida inteira. São Paulo: Editorial Pensamento; 2002, 24-33.

14.BENETTON J. Hábitos, cotidiano e terapia ocupacional. Revista do centro de estudos de terapia ocupacional,2003;8:17-32.

15.PARCKER M. A senda do Yoga . Filosofia,Prática e Terapêutica. Brasília: Editorial Teosófica;2008, 87-94.

16.SOUZA C. Saúde coletiva e Corporeidade - do exercício físico à ecologia humana. Dynamis,Revista Tecno - Cientifica. 2004;49: 25-47.

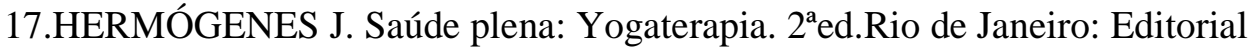

Record;1992,67-75.

18.ALEMAIDA M. Corpo e arte em terapia ocupacional. Rio de Janeiro: Editorial Enelivros;2004,32-44. 